\title{
Response to Clark and Kupper
}

read with great interest the commentary by Clark and Kupper titled Misbehaving macrophages in the pathogenesis of psoriasis (1) regarding our recently published articles in the JCI $(2,3)$. I very much appreciate this instructive commentary, but I feel that it should be put in perspective, in particular for readers who are not specialists in the field of psoriasis.

First, to date, only 2 of the 8 mouse models cited in the commentary have shown conclusively that $\mathrm{T}$ cells are required in order for the psoriasis-like skin phenotype to develop in the respective model. In these 2 models, elimination of human $\mathrm{CD}^{+} \mathrm{T}$ cells from transplanted skin of 3 psoriatic individuals (4) or depletion of $\mathrm{CD}^{+} \mathrm{T}$ cells from CD18 hypomorphic mice (5) led to an improvement of the phenotype. Three of the other mouse models have shown that injection of in vitro-activated $\mathrm{T}$ cells $/ \mathrm{CD} 4^{+} \mathrm{T}$ cells into skin grafts $(6,7)$ or of MHC-mismatched $\mathrm{CD}^{+} \mathrm{T}$ lymphocytes into SCID mice (8) reproduces or produces a psoriasis-like skin phenotype. This indicates that $\mathrm{T}$ cells can be sufficient but does not prove that they are required for the development of the psoriasis-like changes in the skin.

Second, not all psoriasis patients respond sufficiently to cyclosporine A treatment. The amenability of psoriasis to cyclosporine treatment is used as an argument in favor of a pathogenic function of T cells. Although cyclosporine A is one of the most efficient drugs in the treatment of psoriasis, trials and clinical experience indicate that a subset of patients does not sufficiently respond to this treatment. For example, a meta-analysis of 3 controlled studies in 597 patients with severe psoriasis (9) demon- strated that only about half of the patients achieved a $70 \%$ or better improvement of their skin symptoms with cyclosporine A. Moreover, cyclosporine A not only affects T cells but also affects many other cell types, e.g., monocytes/macrophages, in which it can inhibit the production of TNF- $\alpha$ (10), a cytokine with a known function in the development of psoriasis.

Third, the T cell-depleting agent denileukin diftitox (ONTAK) has poor efficacy in the treatment of psoriasis. $\mathrm{DAB}_{389} \mathrm{IL}-2$ is a fusion protein consisting of IL-2 fused to diphtheria toxin. It binds to cells expressing the high-affinity IL-2 receptor and causes their destruction. In a double-blind, placebo-controlled phase II multicenter trial in patients with psoriasis, the efficacy of $\mathrm{DAB}_{389} \mathrm{IL}-2$ as compared with placebo was minimal, and no dose-response relationship could be established (11).

Finally, although it is likely that $\mathrm{T}$ lymphocytes have important functions in the development of psoriasis, it seems too early to conclude upon their pathogenic role. While a contribution of $\mathrm{T}$ cells may be critical for the development of psoriasis in a number of cases, any generalization of this concept would be premature at present. Instead, it should be considered that pathogenic pathways leading to the multifaceted clinical picture of psoriasis could be heterogeneous and may not necessarily depend on 1 single cell type.

\section{Ingo Haase}

Department of Dermatology, University of Cologne, Cologne, Germany.

Conflict of interest: The author has declared that no conflict of interest exists.
Address correspondence to: Ingo Haase, Department of Dermatology and Center for Molecular Medicine, University of Cologne (CMMC), Joseph-StelzmannStrasse 9, 50924 Cologne, Germany. Phone: 49-221-478-86360; Fax: 49-221-478-5949; E-mail: Ingo.Haase@uni-koeln.de.

J. Clin. Invest. 116:3088 (2006). doi:10.1172/ JCI30698.

1. Clark, R.A., and Kupper, T.S. 2006. Misbehaving macrophages in the pathogenesis of psoriasis. J. Clin. Invest. 116:2084-2087. doi:10.1172/JCI29441.

2. Stratis, A., et al. 2006. Pathogenic role for skin macrophages in a mouse model of keratinocyteinduced psoriasis-like skin inflammation. J. Clin. Invest. 116:2094-2104. doi:10.1172/JCI27179.

3. Wang, H., et al. 2006. Activated macrophages are essential in a murine model for $\mathrm{T}$ cell-mediated chronic psoriasiform skin inflammation. J. Clin. Invest. 116:2105-2114. doi:10.1172/JCI27180.

4. Boyman, O., et al. 2004. Spontaneous development of psoriasis in a new animal model shows an essential role for resident $\mathrm{T}$ cells and tumor necrosis factoralpha. J. Exp. Med. 199:731-736.

5. Kess, D., et al. 2003. CD4+ T cell-associated pathophysiology critically depends on CD18 gene dose effects in a murine model of psoriasis. J. Immunol. 171:5697-5706.

6. Sano, S., et al. 2005. Stat3 links activated keratinocytes and immunocytes required for development of psoriasis in a novel transgenic mouse model. Nat. Med. 11:43-49.

7. Nickoloff, B.J., and Wrone-Smith, T. 1999. Injection of pre-psoriatic skin with CD4+ T cells induces psoriasis. Am. J. Pathol. 155:145-158.

8. Schon, M.P., Detmar, M., and Parker, C.M. 1997. Murine psoriasis-like disorder induced by naive CD4+ T cells. Nat. Med. 3:183-188.

9. Faerber, L., et al. 2001. Cyclosporine in severe psoriasis. Results of a meta-analysis in 579 patients. Am. J. Clin. Dermatol. 2:41-47.

10. Nguyen, D.T., et al. 1990. Cyclosporin a modulation of tumor necrosis factor gene expression and effects in vitro and in vivo. J. Immunol. 144:3822-3828.

11. Bagel, J., et al. 1998. Administration of DAB389IL-2 to patients with recalcitrant psoriasis: a doubleblind, phase II multicenter trial. J. Am. Acad. Dermatol. 38:938-944.

\section{Response to Field}

W tary (1) accompanying our article (2) in the October issue of the JCI. We appreciate Michael Field's opinion that based on our data, the concept of secretory diarrhea must be revised to include the idea that net water secretion, or diarrhea, can occur in the absence of active ion secretion. How- ever, we disagree with his conclusion that TNF must affect capillary permeability or smooth muscle contractility to generate increased interstitial pressure and a hydraulic driving force for water secretion.

In contrast to Field's suggestion, our study of 2 TNF superfamily members, TNF and LIGHT (lymphotoxin-like inducible protein that competes with glycoprotein $\mathrm{D}$ for herpesvirus entry mediator on $\mathrm{T}$ cells), demonstrates that water secretion occurs as a consequence of epithelial $\mathrm{Na}^{+}$ malabsorption and increased epithelial paracellular permeability. This is supported by the observation that although TNF and LIGHT cause quantitatively similar para- 
cellular permeability increases, only TNF causes diarrhea. The key difference that accounts for this is that only TNF activates PKC $\alpha$, thereby inhibiting $\mathrm{Na}^{+} / \mathrm{H}^{+}$exchanger isoform 3 (NHE3); TNF did not induce diarrhea when PKC $\alpha$-mediated NHE3 inhibition was prevented by either pharmacological inhibitors or PKC $\alpha$ knockout. Could these approaches to PKC $\alpha$ inhibition have also prevented PKC $\alpha$-dependent changes in capillary permeability or smooth muscle contractility? To test this we took advantage of the observation that LIGHT does not activate PKC $\alpha$. LIGHT did cause diarrhea when PKC was activated pharmacologically, confirming that LIGHT was fully able to recapitulate TNF-like diarrhea when coupled with PKC activation. More importantly, direct NHE3 inhibition, either pharmacological or genetic, showed that this was the critical second component necessary for LIGHT to cause diarrhea. This was not due to inhibition of endothelial or smooth muscle NHE3, since neither tissue expresses NHE3. The unmistakable conclusion is that the necessary role of PKCa in TNF-induced diarrhea is to inhibit epithelial NHE3 and not to effect changes in capillary permeability or smooth muscle contractility.

Why, then, is NHE3 so critical? Is it simply that $\mathrm{Na}^{+}$absorption provides an osmotic driving force for water absorption? This alone is an inadequate explanation, as inhibition of absorption is not necessarily equivalent to induction of secretion. We propose that loss of NHE3-mediated $\mathrm{Na}^{+}$ absorption results in dissipation of the normal hyperosmolarity of the upper villus that drives water absorption (3). It then fol- lows that increased paracellular permeability allows $\mathrm{Na}^{+}$to leak from tissue to lumen; without $\mathrm{NHE} 3, \mathrm{Na}^{+}$cannot be reabsorbed. Increased epithelial paracellular permeability may also allow water and other solutes to flow into the lumen, thereby compounding loss of the villous osmotic gradient that drives water absorption. In terms of absorption, the model gains further support from the observation that LIGHTinduced increases in permeability enhance water absorption (when NHE3 is active). Thus, this model fully explains the data without the need to invoke increased interstitial pressure to force water through paracellular or transcellular water channels.

Is it therefore impossible for elevated interstitial pressure, secondary to increased capillary permeability or smooth muscle contraction, to contribute to TNF-induced diarrhea? It is not. However, if elevated venous or interstitial pressure were able to cause diarrhea by the mechanism proposed by Yablonski and Lifson (4) that is cited by Field (1), it is surprising that diarrhea is not a common clinical characteristic of patients with portal hypertension and that jejunal water and electrolyte transport are normal in these patients (5).

We therefore conclude that TNF causes acute diarrhea via myosin light chain kinasedependent increases in epithelial paracellular permeability coupled with PKC $\alpha$-dependent NHE3 inhibition. Although intriguing as a hypothesis, no data support the role of increased interstitial pressure physically squeezing water into the lumen as proposed by Field. However, existing models of epithelial transport do provide an ample explanation for the observed synergy between increased epithelial paracellular permeability and $\mathrm{Na}^{+}$malabsorption.

Jerrold R. Turner, ${ }^{1}$ Daniel R. Clayburgh, ${ }^{1}$ Mark W. Musch, ${ }^{2}$ Michael Leitges, ${ }^{3}$ and Yang-Xin Fu ${ }^{1}$

${ }^{1}$ Department of Pathology and ${ }^{2}$ Department of Medicine, The University of Chicago, Chicago, Illinois, USA. ${ }^{3}$ Department of Nephrology, Hannover Medical School, Hannover, Germany.

Conflict of interest: The authors have declared that no conflict of interest exists.

Address correspondence to: Jerrold Turner, The University of Chicago, 5841 South Maryland, MC 1089, Chicago, Illinois 60637, USA. Phone: (773) 702-2433; Fax: (773) 8345251; E-mail: jturner@bsd.uchicago.edu.

J. Clin. Invest. 116:3088-3089 (2006). doi:10.1172/JCI30738.

1. Field, M. 2006. T cell activation alters intestinal structure and function. J. Clin. Invest. 116:2580-2582. doi:10.1172/JCI29985.

2. Clayburgh, D.R., Musch, M.W., Leitges, M., Fu, Y.-X., and Turner, J.R. 2006. Coordinated epithelial NHE3 inhibition and barrier dysfunction are required for TNF-mediated diarrhea in vivo. J. Clin. Invest. 116:2682-2694. doi:10.1172/JCI29218.

3. Hallback, D.A., Jodal, M., Mannischeff, M., and Lundgren, O. 1991. Tissue osmolality in intestinal villi of four mammals in vivo and in vitro. Acta Physiol. Scand. 143:271-277.

4. Yablonski, M.E., and Lifson, N. 1976. Mechanism of production of intestinal secretion by elevated venous pressure. J. Clin. Invest. 57:904-915.

5. Norman, D.A., Atkins, J.M., Seelig, L.L., Jr., GomezSanchez, C., and Krejs, G.J. 1980. Water and electrolyte movement and mucosal morphology in the jejunum of patients with portal hypertension. Gastroenterology. 79:707-715. 